\title{
Adoption of Improved Chickpea Production Technology in Haryana
}

\author{
Nidhi Sharma ${ }^{*}$, P.S. Shehrawat ${ }^{1}$, Amit Kumar ${ }^{1}$ and Aditya ${ }^{2}$ \\ ${ }^{1}$ Department of Extension Education, CCSHAU, Hisar, Haryana, India \\ ${ }^{2} \mathrm{CoA}$, CCSHAU, Hisar, Haryana, India \\ *Corresponding author: psshehrawat1965@gmail.com (ORCID ID: 0000-0001-7740-6680)
}

Received: $10-01-2020$

Revised: $20-04-2020$

Accepted: 27-05-2020

\begin{abstract}
Chickpea is one of the important crop among pulses. It is important proteinaceous diet especially for vegetarian people. The total sample comprising of 120 chickpea growers as respondents from four villages in Tosham and Siwani block of Bhiwani district were selected. The data shows that majority of respondents used HC-3 variety (47\%) followed by C-235 (41.67\%) and HC-5 (33.33\%). Maximum respondents (98\%) had high level of adoption followed by 68.00 per cent respondents had low level of adoption in seed rate. Majority of respondents (96.66 \%) had high level of adoption followed by 95.00 per cent had medium level of adoption and 91.66 per cent had low level of adoption regarding field preparation. Maximum respondents $(97 \%$ ) had high level of adoption followed by 92.33 per cent had medium level of adoption and 33.33 per cent had low level of adoption in case of sowing time. Most of the respondents (69.33\%) applied Di-ammonium phosphate as fertilizer followed by urea (66.66 \%), single super phosphate (36\%) and zinc sulphate (33.33\%), respectively. Majority of respondents (67.33\%) applied irrigation before flowering (45-60 days after sowing) followed by (33.33\%) respondents applied irrigation at pod formation stage and 33.33 per cent adopted seed treatment with Bavistin or Trichodermma viridae for wilt control. Similarly, 33.33 per cent respondents adopted cultural control of diseases (Ascochyta blight, Alternaria blight and Grey mold).
\end{abstract}

\section{Highlights}

(0 The main focus of study is to find out adoption of improved chickpea production technology.

Keywords: Adoption, chickpea, recommended cultivation, rhizobium, seed treatment

Chickpea (Cicer arietinum L.) is also known as gram which is one of the important pulse crop of our country. It is a protein-rich diet especially to the poor in developing countries, where people are vegetarians or cannot afford animal protein and helps in enhancing the soil quality for subsequent cereal crop cultivation. India is the largest producer of chickpea but still imports chickpea from other countries and ranked first in area and production of chickpea in the world, followed by Pakistan, Australia and Iran. The highest productivity of chickpea $(6120 \mathrm{~kg} / \mathrm{ha})$ is observed in Isreal followed by Yemen, Canada and Egypt and it is second most important pulse growing country worldwide in terms of area under cultivation but ranks third in production. At world level, total area of pulses in 2016 was 54.23 million hectares. Production of pulses was 39.66 million tonnes with $731 \mathrm{~kg} /$ hectare yield. In 2016-17 production of total pulses in India was 29280 thousand hectares with 22950 thousand tonne productions (Anonymous, 2017).

Madhya Pradesh, Rajasthan, Maharashtra, Uttar Pradesh, Andhra Pradesh, Karnataka, Chhattisgarh, Bihar and Jharkhand are major chickpea producing

How to cite this article: Sharma, N., Shehrawat, P.S. and Kumar, A. and Aditya (2020). Adoption of improved chickpea production technology in Haryana. Economic Affairs, 65(2): 183-189.

Source of Support: None; Conflict of Interest: None 
AESSRA

Sharma et al.

states contributing more than 95 per cent to the total chickpea production. Madhya Pradesh is the single largest producer in the country accounting for over 40 per cent of total production while Rajasthan, Maharashtra, Uttar pradesh and Andhra pradesh contribute about 14 per cent, 10 per cent, 9 per cent and 7 per cent, respectively.

It was observed that the productivity of chickpea was found low in comparison to their potential yield in existing area. It showed improvement in chickpea production was needed through conservation, diversification of agriculture and to enhance adoption level of improved chickpea production technology. So as to increase the productivity, particularly under rainfed chickpea growing regions was one of the major challenges and concern which need to be addressed on priority basis. A need has been felt to identify the gap in technology adoption in pulse crops so that appropriate measure can be taken to emphasize the adoption of improved technology, which will ultimately lead to increased productivity and production. Keeping these things in mind, the objectives of study was undertaken to know the personal profile of chickpea growers and to find out the adoption level of improved chickpea production technology.

\section{METHODOLOGY}

The study was conducted in Bhiwani district of Haryana. Two blocks Tosham and Siwani were selected from Bhiwani district. Two villages were randomly selected from each block. Thus, total number of four villages were selected, namely, Khanak and Kirawar from Tosham, and Gaindawas and Barwa from Siwani. From each village, 30 farmers were selected randomly. Thus, the total numbers of 120 farmers were interviewed. The data collected were tabulated and analyzed. The appropriate statistical tools i.e. percentage, total weighted score, mean score, rank order were used. Rank order was calculated by aggregate total score were calculated based on calculated score. A weighted mean score was obtained and the maximum weighted mean score so obtained were given the rank $1^{\text {st }}$ and the next subsequent one was given $2^{\text {nd }}$ and so on the descending orders.

Adoption is operationally defined as degree of actual use of improved cultivation practices by Gram growers. It was measured on three point continuum as fully adopted, partially adopted and somewhat adopted by assigning the score of 3, 2 and 1, respectively. The adoption score was then converted into adoption index by applying following formula:

Adoption index $=$

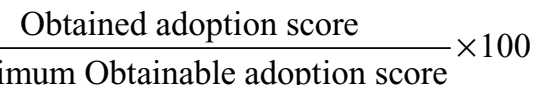

Later on respondents were categorized as fully adopted, partially adopted and somewhat adopted, respectively.

\section{RESULTS AND DISCUSSION}

The collected information was analyzed for ascertaining level of adoption of recommended technology in chickpea cultivation.

The data reported in table 1 reveals that the majority of respondents (39.16 per cent) were matriculate, followed by 25.83 percent, 14.16 per cent, 10.83 per cent. 5.83 per cent, and 4.16 per cent and 0.00 per cent was having education middle school, higher secondary, primary, graduate, illiterate and postgraduate. It was also observed that two third of respondents (63.33 per cent) were living in nuclear family system and the remaining (36.66 per cent) respondents were living in joint family system in the area of investigation. It has been also depicted that respondents It is apparent from table 1 that 70 per cent respondents were engaged in farming and only 50 per cent of the farmers had job or other business along with farming.

Table 1: Profile of chickpea growers $(\mathrm{N}=120)$

\begin{tabular}{|c|c|c|c|c|}
\hline $\begin{array}{l}\text { Sl. } \\
\text { No. }\end{array}$ & $\begin{array}{l}\text { Variable } \\
\text { (s) }\end{array}$ & Category & Number & Percentage \\
\hline \multirow{3}{*}{1} & \multirow{3}{*}{ Age } & Young (20-35 years) & 29 & 24.16 \\
\hline & & Middle(36-50 years) & 49 & 40.83 \\
\hline & & Old (above 50 years) & 42 & 35.00 \\
\hline \multirow{7}{*}{2} & \multirow{7}{*}{ Education } & Illiterate & 05 & 04.16 \\
\hline & & Primary & 13 & 10.83 \\
\hline & & Middle & 31 & 25.83 \\
\hline & & Metric & 47 & 39.16 \\
\hline & & Higher secondary & 17 & 14.16 \\
\hline & & Graduate & 07 & 05.83 \\
\hline & & Post graduate & 00 & 0.00 \\
\hline
\end{tabular}




\begin{tabular}{|c|c|c|c|c|}
\hline \multirow{3}{*}{3} & \multirow{3}{*}{ Caste } & General & 26 & 21.66 \\
\hline & & OBC & 64 & 53.33 \\
\hline & & SC & 30 & 25.00 \\
\hline \multirow{2}{*}{4} & \multirow{2}{*}{$\begin{array}{l}\text { Family } \\
\text { type }\end{array}$} & Nuclear & 76 & 63.33 \\
\hline & & Joint & 44 & 36.66 \\
\hline \multirow{3}{*}{5} & \multirow{3}{*}{$\begin{array}{l}\text { Family } \\
\text { size }\end{array}$} & $\begin{array}{l}\text { Small (up to } 4 \\
\text { members ) }\end{array}$ & 40 & 33.00 \\
\hline & & $\begin{array}{l}\text { Medium ( } 5 \text { to } 6 \\
\text { members) }\end{array}$ & 52 & 43.33 \\
\hline & & $\begin{array}{l}\text { Large ( } 7 \text { members } \\
\text { and above) }\end{array}$ & 28 & 23.33 \\
\hline \multirow[b]{2}{*}{6} & \multirow{2}{*}{$\begin{array}{l}\text { Occupa- } \\
\text { tion }\end{array}$} & Only farming & 70 & 58.33 \\
\hline & & $\begin{array}{l}\text { Business/Job and } \\
\text { farming }\end{array}$ & 50 & 41.66 \\
\hline \multirow{6}{*}{7} & \multirow{6}{*}{$\begin{array}{l}\text { Land } \\
\text { holding }\end{array}$} & Landless & 00 & 00.00 \\
\hline & & Less than 1 acre & 09 & 07.50 \\
\hline & & $\begin{array}{l}\text { Above } 1 \text { and up to } \\
5 \text { acres }\end{array}$ & 50 & 41.66 \\
\hline & & $\begin{array}{l}\text { Above } 5 \text { and up to } \\
10 \text { acres }\end{array}$ & 29 & 24.16 \\
\hline & & Above 10 to 15 acres & 14 & 11.66 \\
\hline & & Above 15 acres & 18 & 15.00 \\
\hline
\end{tabular}

\section{Status of gram growers of the study area}

\section{Farm implements}

The data presented in table 2, reveals that 100 per cent respondents owned plough, wooden planker, spade and hand hoe whereas 66.60 per cent owned tractor, 62.50 per cent sprayer, 60.83 per cent harrow, 58.33 per cent cultivator, 2.50 per cent seed cum fertilizer drill for using in the agricultural activities.

Table 2: Farm implements available with farmers

$$
\left(\mathrm{N}=120^{*}\right)
$$

\begin{tabular}{clcc}
\hline S1. No. & Farm implements & Frequency & Percentage \\
\hline 1 & Tractor & 80 & 66.60 \\
2 & Harrow & 73 & 60.83 \\
3 & Cultivator & 70 & 58.33 \\
4 & Seed cum fertilizer drill & 03 & 02.50 \\
5 & Laser land leveler & 00 & 00.00 \\
6 & Combined machine & 00 & 00.00 \\
7 & Puddler & 00 & 00.00 \\
8 & Rotavator & 00 & 00.00 \\
9 & Plough & 120 & 100.00 \\
10 & Wooden planker & 120 & 100.00 \\
11 & Spade & 120 & 100.00 \\
12 & Hand hoe & 120 & 100.00 \\
13 & Sprayer & 75 & 62.50 \\
\hline
\end{tabular}

* Multiple responses.

\section{Cropping system}

The data presented in table 4 reveals that 83.33 per cent respondents had double cropping system followed by 15.00 per cent respondents have monocropping and 1.66 per cent respondents had adopted intercropping.

Table 4: Cropping system $(\mathrm{N}=120)$

\begin{tabular}{clcc}
\hline S1. No. & Cropping system & Frequency & Percentage \\
\hline 1 & Monocropping & 18 & 15.00 \\
2 & Intercropping & 02 & 01.66 \\
3 & Double cropping & 100 & 83.33 \\
\hline & Total & $\mathbf{1 2 0}$ & $\mathbf{1 0 0}$ \\
\hline
\end{tabular}

\section{Crop rotation}

The data mentioned in table 5 indicated that majority of the respondents (85.83 per cent) were growing pearl millet in Kharif season and chickpea in Rabi season followed by 08.33 per cent respondents were growing cotton in Kharif season and chickpea in Rabi season and 05.83 per cent respondents were growing sorghum in Kharif season and chickpea in Rabi season.

Table 5: Crop rotation $(\mathrm{N}=120)$

\begin{tabular}{|c|c|c|c|c|}
\hline S1. No. & Kharif & Rabi & Frequency & Percentage \\
\hline 1 & Pearl millet & Chick pea & 103 & 85.83 \\
\hline 2 & Maize & Chick pea & 00 & 00.00 \\
\hline 3 & Sorghum & Chick pea & 07 & 05.83 \\
\hline \multirow[t]{2}{*}{4} & Cotton & Chick pea & 10 & 08.33 \\
\hline & Total & & 120 & 100 \\
\hline
\end{tabular}

\section{Irrigation facilities}

The sources of irrigation and assured irrigation are one of the most important factors for agriculture. It can be observed that farmers were having tube wells; canal and submersible pump for irrigation, which is, own and hired.

Table 6: Irrigation facilities ( $\mathrm{N}=120)$

\begin{tabular}{clcc}
\hline S1. No. & Modes of irrigation & Frequency & Percentage \\
\hline 1 & Submersible pump & 06 & 05.00 \\
2 & Tube Well (own) & 18 & 15.00 \\
3 & Tube Well (hire basis) & 52 & 43.33 \\
4 & Canal & 44 & 36.66 \\
\hline & Total & $\mathbf{1 2 0}$ & $\mathbf{1 0 0 . 0 0}$ \\
\hline
\end{tabular}


It is clear from table 6 revealed that 15.00 per cent respondents are using tube well which is owned and 43.33 per cent hired as irrigation sources followed by 36.66 per cent respondents used canal water for irrigation and 5.00 per cent respondents used submersible pump as irrigation source.

\section{Mass media exposure}

The data in table 7 revealed that reading of newspaper ranked first with mean score of 2.85, followed by TV and radio ranked second and third with weighted mean score of 2.83 and 2.10, respectively. Kisan Seva Kendra was ranked fourth with weighted mean score of 1.43 , magazine was ranked fifth with weighted mean score of 0.85 and internet was ranked last with 0.43 as weighted mean score, whereas only 45.83 per cent of farmers obtained training.

\section{Extension contact}

This section comprises of contact of farmers with various extension officials and the frequency of their contact. Various extension officials with whom the farmers had contacts are enlisted in the table 8 with the frequency of their contacts.

The data depicted in table 8 revealed that among the extension contact of farmers, the most popular were the progressive farmers with weighted mean score 3.80. Scientists and ADO ranked second and third among extension officials with weighted mean score 2.85 and 2.30, followed by SDAO/SMS and NGO as fourth and fifth with weighted mean score 1.98 and 0.26 , respectively.

It is evident from the table 9 regarding varieties, HC-3 had high level of adoption (47 per cent) followed by C-235 had medium level of adoption (41.67 per cent) and HC-5 had low level of adoption (33.33 per cent).

In case of seed rate, 98.00 per cent respondents had high level of adoption followed by 68.00 per cent respondents had low level of adoption.

With regard to seed treatment/ use of Rhizobium culture were found that majority of respondents had 33.30 per cent level of adoption at the time of investigation.

Table 7: Mass media exposure $(\mathrm{N}=120)$

\begin{tabular}{|c|c|c|c|c|c|c|c|c|}
\hline \multirow[b]{2}{*}{$\begin{array}{l}\text { Sl. } \\
\text { No. }\end{array}$} & \multirow[b]{2}{*}{ Mass media } & \multirow[b]{2}{*}{ Used } & \multicolumn{3}{|c|}{ Extent of utilization } & \multirow[b]{2}{*}{$\begin{array}{l}\text { - Total } \\
\text { score }\end{array}$} & \multirow[b]{2}{*}{$\begin{array}{l}\text { Weighted } \\
\text { mean score }\end{array}$} & \multirow[b]{2}{*}{ Rank } \\
\hline & & & $\begin{array}{l}\text { Daily } \\
\text { (3) }\end{array}$ & $\begin{array}{l}\text { Often } \\
\text { (2) }\end{array}$ & $\begin{array}{l}\text { Sometimes } \\
\text { (1) }\end{array}$ & & & \\
\hline 1 & Newspaper & $118(98.33)$ & $109(327)$ & 07(14) & $02(2)$ & 343 & 02.85 & I \\
\hline 2 & TV & $116(96.67)$ & $110(330)$ & $04(8)$ & $02(2)$ & 340 & 02.83 & II \\
\hline 3 & Radio & $94(78.33)$ & $70(210)$ & 19(38) & $05(5)$ & 253 & 02.10 & III \\
\hline 4 & Kisan Seva Kendra & $100(83.33)$ & $03(9)$ & $66(132)$ & $31(31)$ & 172 & 01.43 & IV \\
\hline 5 & Magazine & $55(45.83)$ & $12(36)$ & $23(46)$ & $20(20)$ & 102 & 00.85 & $\mathrm{~V}$ \\
\hline 6 & Internet & $21(17.5)$ & $13(39)$ & $05(10)$ & $03(3)$ & 52 & 00.43 & VI \\
\hline 7 & Workshop/Training & $55(45.83)$ & - & - & - & - & - & - \\
\hline
\end{tabular}

(Figures in parentheses in column 3 indicate percentages; columns 8 indicate weighted mean scores and column 9 indicates ranks).

Table 8: Extension contact $(\mathrm{N}=120)$

\begin{tabular}{|c|c|c|c|c|c|c|c|c|c|}
\hline \multicolumn{10}{|c|}{ Frequency of contact } \\
\hline $\begin{array}{l}\text { Sl. } \\
\text { No. }\end{array}$ & $\begin{array}{l}\text { Extension } \\
\text { Official }\end{array}$ & $\begin{array}{l}\text { Weekly } \\
\text { (4) }\end{array}$ & $\begin{array}{l}\text { Fortnightly } \\
\text { (3) }\end{array}$ & $\begin{array}{l}\text { Monthly } \\
\text { (2) }\end{array}$ & $\begin{array}{l}\text { Whenever } \\
\text { Needed } \\
\text { (1) }\end{array}$ & $\begin{array}{l}\text { None } \\
(0)\end{array}$ & $\begin{array}{l}\text { Total } \\
\text { score }\end{array}$ & $\begin{array}{l}\text { Weighted } \\
\text { mean score }\end{array}$ & Rank \\
\hline 1 & Progressive farmer & $110(440)$ & $4(12)$ & $1(2)$ & $3(3)$ & $2(0)$ & 457 & 03.80 & I \\
\hline 2 & Scientists & $64(256)$ & $14(42)$ & $10(20)$ & $24(24)$ & $8(0)$ & 342 & 02.85 & II \\
\hline 3 & $\mathrm{ADO}$ & $44(176)$ & $8(24)$ & $13(26)$ & $51(51)$ & $4(0)$ & 277 & 02.30 & III \\
\hline 4 & SDAO/SMS & $30(120)$ & $13(39)$ & $9(18)$ & $60(60)$ & $8(0)$ & 237 & 01.98 & IV \\
\hline 5 & NGO & $5(20)$ & $1(3)$ & $3(6)$ & $2(2)$ & $109(0)$ & 31 & 00.26 & $\mathrm{~V}$ \\
\hline
\end{tabular}

(Figures in parentheses indicates percentage; columns 5 indicates weighted mean scores and column 6 indicates ranks) 
Table 9: Adoption of recommended chickpea production practices

\begin{tabular}{|c|c|c|c|c|c|c|c|c|}
\hline \multicolumn{9}{|c|}{ Varieties } \\
\hline $\begin{array}{l}\text { Sl. } \\
\text { No. }\end{array}$ & Recommended varieties & $\begin{array}{l}\text { Fully } \\
\text { Adopted } \\
\text { (3) }\end{array}$ & $\begin{array}{l}\text { Partially } \\
\text { Adopted } \\
\text { (2) }\end{array}$ & $\begin{array}{l}\text { Somewhat } \\
\text { adopted } \\
\text { (1) }\end{array}$ & $\begin{array}{l}\text { Total } \\
\text { mean } \\
\text { score }\end{array}$ & $\begin{array}{l}\text { Weighted } \\
\text { mean score }\end{array}$ & Percentage & $\begin{array}{l}\text { Rank } \\
\text { order }\end{array}$ \\
\hline \multicolumn{9}{|c|}{ For Desi Chickpea } \\
\hline 1 & C-235 & $5(15)$ & $20(40)$ & 95(95) & 150 & 1.25 & 41.67 & II \\
\hline 2 & HC-1 & $11(33)$ & $49(98)$ & $60(60)$ & 191 & 1.59 & 38.66 & IV \\
\hline 3 & HC-3 & $0(0)$ & $50(100)$ & $70(70)$ & 170 & 1.41 & 47.00 & I \\
\hline 4 & HC-5 & $0(0)$ & $0(0)$ & $120(120)$ & 120 & 1.00 & 33.33 & VI \\
\hline \multicolumn{9}{|c|}{ For Kabuli Chickpea } \\
\hline 5 & HK-1 & $5(15)$ & $10(20)$ & 105(105) & 140 & 1.16 & 38.00 & $\mathrm{~V}$ \\
\hline 6 & HK-2 & $5(15)$ & $15(30)$ & $100(100)$ & 145 & 1.20 & 40.00 & III \\
\hline & Seed rate & & & & & & & \\
\hline 1 & Desi Chickpea (15-18 kg/acre) & 113(339) & $7(14)$ & $0(0)$ & 353 & 2.94 & 98.00 & I \\
\hline 2 & Kabuli Chickpea (36kg/acre) & $10(30)$ & $5(10)$ & 105(105) & 245 & 2.04 & 68.00 & II \\
\hline \multicolumn{9}{|c|}{ Seed treatment } \\
\hline 1 & $\begin{array}{l}\text { Treatment with Rhizobium } \\
\text { culture }\end{array}$ & $0(0)$ & $0(0)$ & $120(120)$ & 120 & 1 & 33.33 & I \\
\hline \multicolumn{9}{|c|}{ Preparatory tillage } \\
\hline 1 & $\begin{array}{l}\text { Number of ploughing done } \\
\text { during last year }\end{array}$ & $112(336)$ & $8(16)$ & $0(0)$ & 342 & 2.85 & 95.00 & I \\
\hline 2 & $\begin{array}{l}\text { Name the plough used for } \\
\text { ploughing }\end{array}$ & 109(327) & $11(22)$ & $0(0)$ & 369 & 3.07 & 96.66 & II \\
\hline 3 & $\begin{array}{l}\text { Number of the planking done } \\
\text { after ploughing }\end{array}$ & $90(270)$ & $30(60)$ & $0(0)$ & 330 & 2.75 & 91.66 & III \\
\hline \multicolumn{9}{|c|}{ Sowing time } \\
\hline 1 & $\begin{array}{l}\text { Around mid October (Desi } \\
\text { chickpea) }\end{array}$ & 112(336) & $6(12)$ & $2(2)$ & 350 & 2.91 & 97.00 & $\mathrm{I}$ \\
\hline 2 & $\begin{array}{l}\text { Around last week of October } \\
\text { (Kabuli chickpea) }\end{array}$ & $95(285)$ & $23(46)$ & $2(2)$ & 333 & 2.77 & 92.33 & II \\
\hline 3 & $\begin{array}{l}2^{\text {nd }} \text { week of November to } 3^{\text {rd }} \\
\text { week of Nov (For irrigated } \\
\text { conditions) }\end{array}$ & $0(0)$ & $0(0)$ & $120(120)$ & 120 & 1.00 & 33.33 & III \\
\hline \multicolumn{9}{|c|}{ Sowing Method } \\
\hline 1 & $\begin{array}{l}\text { Method of sowing } \\
\text { (Line sowing, Seed drill and } \\
\text { Pora method) }\end{array}$ & $43(129)$ & $67(134)$ & & 273 & 2.27 & 75.66 & I \\
\hline 2 & $\begin{array}{l}\text { Spacing at yours } \\
\text { farm }(R \times R=45 \mathrm{~cm})\end{array}$ & $33(99)$ & $70(140)$ & & 256 & 2.13 & 71.00 & II \\
\hline \multicolumn{9}{|c|}{ Manures and fertilizers } \\
\hline 1 & Urea $(12 \mathrm{~kg} / \mathrm{ac})$ & $32(96)$ & $56(112)$ & $32(32)$ & 240 & 2.00 & 66.66 & II \\
\hline 2 & SSP (100kg/ac) & $0(0)$ & $10(20)$ & $110(110)$ & 130 & 1.08 & 36.00 & III \\
\hline 3 & $\mathrm{DAP}(35 \mathrm{~kg} / \mathrm{ac})$ & $21(63)$ & $88(176)$ & $11(11)$ & 250 & 2.08 & 69.33 & I \\
\hline 4 & $\mathrm{ZnSo}_{4}(10 \mathrm{~kg} / \mathrm{ac})$ & $0(0)$ & $0(0)$ & $120(120)$ & 120 & 1.00 & 33.33 & IV \\
\hline \multicolumn{9}{|c|}{ Irrigation Requirement } \\
\hline 1 & Before flowering & $5(15)$ & $113(226)$ & $2(2)$ & 243 & 2.02 & 67.33 & $\mathrm{I}$ \\
\hline 2 & Pod formation stage & $0(0)$ & $0(0)$ & $120(120)$ & 120 & 1.00 & 33.33 & II \\
\hline
\end{tabular}




\begin{tabular}{|c|c|c|c|c|c|c|c|c|c|c|}
\hline \multicolumn{11}{|c|}{ Weed control } \\
\hline 1 & 25-30DAS & & 116(348) & $4(8)$ & & $0(0)$ & 356 & 2.96 & 98.66 & I \\
\hline 2 & 45-50DAS & & $23(69)$ & $50(10$ & & $4(4)$ & 173 & 1.44 & 48.00 & II \\
\hline \multicolumn{11}{|c|}{ Disease control } \\
\hline 1 & $\begin{array}{l}\text { Wilt (Seed tre } \\
\text { Trichodermma } \\
\text { vitavax } 1 \mathrm{~g} / \mathrm{kg}\end{array}$ & $\begin{array}{l}\text { ment with } \\
\text { ridae } 4 \mathrm{~g} / \mathrm{kg} \text { plus }\end{array}$ & $0(0)$ & $0(0)$ & & $120(120)$ & 120 & 1 & 33.33 & I \\
\hline & $5 \mathrm{ml}$ of water) & & & & & & & & & \\
\hline \multicolumn{11}{|c|}{ Cultural control } \\
\hline 1 & $\begin{array}{l}\text { Ascochyta } \\
\text { blight }\end{array}$ & Grow resistant & tolerant & $0(0)$ & $0(0)$ & $120(120)$ & 120 & 1 & 33.33 & $\mathrm{I}$ \\
\hline 2 & $\begin{array}{l}\text { Alternaria } \\
\text { blight }\end{array}$ & $\begin{array}{l}\text { varieties-HC-1 } \\
\text { burn infected } \mathrm{p}\end{array}$ & $\begin{array}{l}\text { and C-235 or } \\
\text { lant parts }\end{array}$ & $0(0)$ & $0(0)$ & 120(120) & 120 & 1 & 33.33 & I \\
\hline 3 & Grey mold & & & $0(0)$ & $0(0)$ & $120(120)$ & 120 & 1 & 33.33 & $\mathrm{I}$ \\
\hline \multicolumn{11}{|c|}{ Chemical insect pest control } \\
\hline 1 & $\begin{array}{l}\text { Gram pod bo } \\
\text { or } 400 \mathrm{~g} \text { carba } \\
\text { crotophos } 36\end{array}$ & $\begin{array}{l}(400 \mathrm{ml} \text { Quinolp } \\
50 \mathrm{WP} \text { or } 200 \mathrm{~m} \\
\text { in } 100 \text { litre of wa }\end{array}$ & $\begin{array}{l}\text { hos } 25 \mathrm{EC} \\
\text { mono- } \\
\text { ter) }\end{array}$ & $0(0)$ & $95(190)$ & $25(25)$ & 215 & 1.79 & 59.66 & I \\
\hline 2 & $\begin{array}{l}\text { Gram dhora } \\
\text { groundnut oi } \\
\text { oil with } 1.75 n\end{array}$ & $\begin{array}{l}\text { e } 7.5 \mathrm{ml} \text { mustar } \\
\mathrm{r} \text { mix mustard o } \\
\text { turmeric } / \mathrm{kg} \text { seed }\end{array}$ & $\begin{array}{l}\text { oil or } \\
\text { groundnut }\end{array}$ & $0(0)$ & $0(0)$ & $120(120)$ & 120 & 1 & 33.33 & II \\
\hline
\end{tabular}

It was observed in preparatory tillage that 96.66 per cent respondents had high level of adoption followed by 95.00 per cent had medium level of adoption and 91.66 per cent had low level of adoption. Regarding sowing time 97.00 per cent respondents had high level of adoption followed by 92.33 per cent had medium level of adoption and 33.33 per cent had low level of adoption. In case of sowing method, 75.66 per cent respondent had high level of adoption followed by 71.00 per cent had low level of adoption. Regarding manures and fertilizers, 69.33 per cent respondents applied Di-ammonium phosphate as fertilizer followed by 66.66 per cent urea, 36.00 per cent single super phosphate and 33.33 per cent zinc sulphate. With regard to irrigation requirement, it was cleared that 67.33 per cent respondents applied irrigation before flowering (45-60 days after sowing) followed by 33.33 per cent respondents applied irrigation at pod formation stage. It was observed in weed control that 98.66 per cent respondents done weeding at 25-30 days after sowing followed by 48 per cent respondents done weeding at 45-50 days after sowing. In case of chemical disease control, 33.33 per cent respondents had adopted chemical control of wilt. Regarding cultural control, 33.33 per cent respondents had adopted cultural control of diseases (Ascochyta blight, Alternaria blight and Grey mould).
In case of chemical insect- pest control, 59.66 per cent respondents had adopted control measure of gram pod borer followed by 33.33 per cent respondents had adopted control measure of gram dhora. It was observed in weed control that 98.66 per cent respondents done weeding at 25-30 days after sowing followed by 48 per cent respondents done weeding at 45-50 days after sowing. In case of chemical disease control, 33.33 per cent respondents had adopted chemical control of wilt. Regarding cultural control, 33.33 per cent respondents had adopted cultural control of diseases (Ascochyta blight, Alternaria blight and Grey mould). In case of chemical insect- pest control, 59.66 per cent respondents had adopted control measure of gram pod borer followed by 33.33 per cent respondents had adopted control measure of gram dhora.

\section{CONCLUSION}

The maximum adoption level was reported in adoption of exact seed rate, preparatory tillage, sowing time and weed control. This was followed by practice like 'method of sowing', 'application of manures and fertilizers', 'irrigation requirement', 'chemical insect-pest control' and 'adoption of improved varieties of chickpea'. While practice like 'seed treatment', 'cultural control', 'chemical 
disease control' were having less adoption level by the respondents.

\section{REFERENCES}

Anonymous. Annual report 2016-17. Directorate of Pulses Development, Ministry of agriculture \& Farmers Welfare. Government of India, 2017.

Badhala, B.S. and Kanojia, Y. 2017. Extent of adoption of improved practices of gram production technology by gram growers in tribal district of Pratapgarh. Agriculture Science Digest, 37(2): 163-165.
Khare, A., Wakle, P.K., Shambharkar, Y.B. and Patil, J. 2013. Correlates of adoption and constraints faced by the gram farmers in adoption of improved cultivation practices. Indian Journal of Research, 2(10): 8- 10.

Singh Beena., Singh Y.K. and Panigrahi, K.T. 2014. Extent of adoption of recommended practices of pulses through first line demonstration. IOSR Journal of Agriculture and Veterinary Science, 7(12): 71-72.

Umrathaiya, K., Choudhary, S. and Swarnakar, V.K. 2015. Comparative study on adoption of improved chickpea varieties in Indore district of M.P. IOSR Journal of Agriculture and Veterinary Science, 8(8): 1-8. 
